\title{
Hardware and first results of TUNKA-HiSCORE
}

\author{
M. Kunnas ${ }^{\mathrm{a}, *}$, M. Brückner ${ }^{\mathrm{c}}$, N. Budnev ${ }^{\mathrm{e}}$, M. Büker ${ }^{\mathrm{a}}$, O. Chvalaev $^{\mathrm{e}}$, A. Dyachok $^{\mathrm{e}}$, \\ U. Einhaus $^{\text {a }, ~ S . ~ E p i m a k h o v ~}{ }^{\text {a }}$, O. Gress ${ }^{e}$, D. Hampf ${ }^{\text {a }}$, D. Horns ${ }^{\text {a }}$, A. Ivanova ${ }^{e}$, \\ E. Konstantinov ${ }^{\text {e, E. Korosteleva }}{ }^{\mathrm{d}}$, L. Kuzmichev ${ }^{\mathrm{d}}$, B. Lubsandorzhiev ${ }^{\mathrm{f}}$, R. Mirgazov ${ }^{\mathrm{e}}$, \\ R. Monkhoev ${ }^{\mathrm{e}}$, R. Nachtigall ${ }^{\mathrm{a}}$, A. Pakhorukov ${ }^{\mathrm{e}}$, V. Poleschuk ${ }^{\mathrm{e}}$, A. Porelli $^{\mathrm{b}}$, V. Prosin ${ }^{\mathrm{d}}$, \\ G.I. Rubtsov $^{\text {f }}$, G.P. Rowell ${ }^{\mathrm{g}}$, P.S. Satunin ${ }^{\mathrm{f}}$, Yu. Semeney ${ }^{\mathrm{e}}$, D. Spitschan ${ }^{\mathrm{a}}$, L. Sveshnikova ${ }^{\mathrm{d}}$, \\ M. Tluczykont ${ }^{\mathrm{a}}, \mathrm{R}$. Wischnewski ${ }^{\mathrm{b}}, \mathrm{A}$. Zagorodnikov ${ }^{\mathrm{e}}$
}

\footnotetext{
a Institute for Experimental Physics, University of Hamburg, Luruper Chaussee 149, 22761 Hamburg, Germany

${ }^{\mathrm{b}}$ DESY, Platanenallee 6, 15738 Zeuthen, Germany

${ }^{\mathrm{c}}$ Institute for Computer Science, Humboldt-University Berlin, Rudower Chaussee 25, 12489 Berlin, Germany

d Skobeltsyn Institute for Nuclear Physics, Lomonosov Moscow State University, 1 Leninskie gory, 119991 Moscow, Russia

e Institute of Applied Physics ISU, Irkutsk, Russia

${ }^{\mathrm{f}}$ Institute for Nuclear Research of the Russian Academy of Sciences 60th October Anniversary st., 7a, 117312 Moscow, Russia

${ }^{\mathrm{g}}$ University of Adelaide 5005, School of Chemistry E' Physics, Australia
}

\section{A R T I C L E I N F O}

Keywords:

HiSCORE

Astroparticle

Non-imaging Cherenkov experiment

\begin{abstract}
A B S T R A C T
As a non-imaging wide-angle Cherenkov air shower detector array with an area of up to $100 \mathrm{~km}^{2}$, the HiSCORE (Hundred*i Square km Cosmic ORigin Explorer) detector concept allows measurements of gamma rays and cosmic rays in an energy range of $10 \mathrm{TeV}$ up to $1 \mathrm{EeV}$. In the framework of the TunkaHiSCORE project we have started measurements with a small prototype array, and planned to build an engineering array $\left(1 \mathrm{~km}^{2}\right)$ on the site of the Tunka experiment in Siberia. The first results and the most important hardware components are presented here.
\end{abstract}

(c) 2013 Elsevier B.V. All rights reserved.

\section{Tunka-HiSCORE}

The Tunka-HiSCORE detector array is a ground-based extensive air shower Cherenkov detector array that samples the air shower light front using the non-imaging technique. With a spacing of about $100-300 \mathrm{~m}$ between stations, it is possible to cover a large area with a small number of detectors. For the physics goals, see Ref. [1], for a description of the expected reconstruction performance see Ref. [2]. We are currently working towards an engineering array [5] of $1 \mathrm{~km}^{2}$ on the site of the Tunka experiment [3,4].

\section{Detector station concept}

\subsection{Planned station setup}

A single detector station consists of four Photomultiplier Tubes (PMTs) of a diameter of 8 in. $(20 \mathrm{~cm})$ with six dynodes that yield a nominal gain of $10^{4}$ at a supply voltage of $1.4 \mathrm{kV}$.

\footnotetext{
* Corresponding author. Tel.: +49 15772704242.

E-mail address: maike.kunnas@desy.de (M. Kunnas).
}

Each PMT is equipped with a light collector (Winston cone) made up of ALANOD 4300 UP material with a reflectivity of $80 \%$. In the standard configuration, the Winston cones have a half opening angle of $30^{\circ}$ and point to the zenith. For a better view of the galactic plane, it is possible to tilt the viewing axis of the stations. The cones increase the light collection area by a factor of four in comparison to pure PMT area, resulting in a total light collection area of $0.5 \mathrm{~m}^{2}$ per station. Each cone is composed of ten segments of reflective material, mounted along the optical axis and fixed with plates at the top and bottom of the cone [2].

To acquire the shower data, the high-gain anode channel signals of the PMTs are split and amplified by a custom fast preamplifier. One set of signals is processed by a clipped sum trigger. In this trigger system, each signal is first clipped at an adjustible clipping threshold, then the clipped signals are summed up. If there was an event, the amplitude of the signal is four times the amplitude of a single clipped signal. The threshold for the following trigger should be slightly below this level so that it only sets off if all four channels had a signal that was high enough to be clipped. This behavior greatly reduces noise, especially the noise produced by afterpulses of the PMTs.

The other set of the PMT signals is summed up and sampled via a custom DRS4 chip based board. For the same purpose we also 


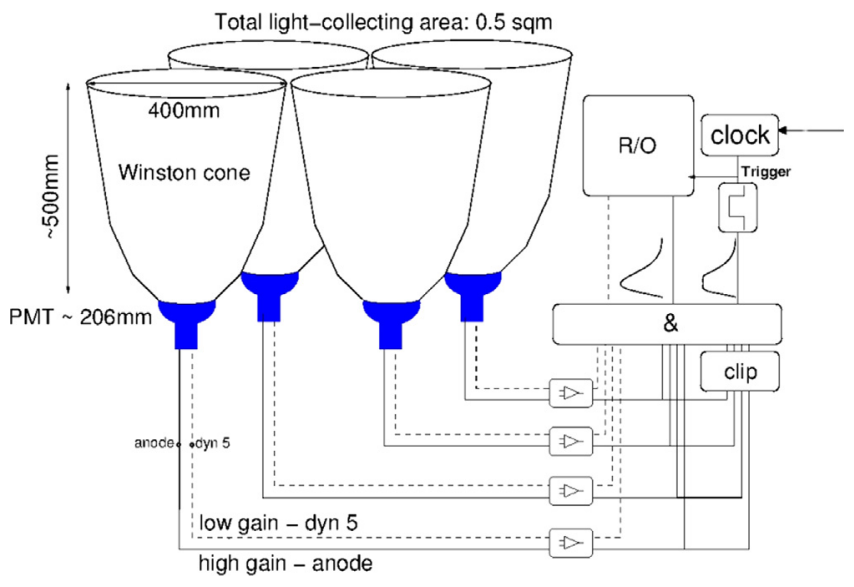

Fig. 1. Concept of a HiSCORE station.

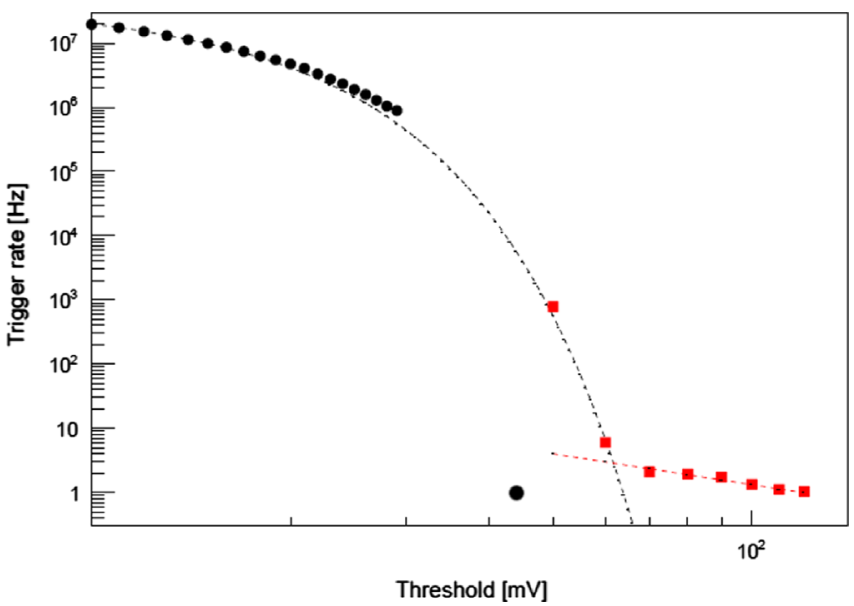

Fig. 2. Comparison between dead-time corrected single station trigger rate (squares) and estimated night sky background spectrum (dots).

sum up and sample the low-gain PMT channels, which are read out at the fifth dynode.

Each event in each station is provided with a time stamp to reconstruct the direction of the shower. For this, the time between stations needs to be synchronised to a nanosecond level (also see Ref. [7]). The system will be based on an improved Tunka time synchronization (based on the carrier frequency of the optical fibers) or on the WhiteRabbit system, a Gbit-ethernet based system developed at CERN for synchronisation of nodes with a spacing of up to $10 \mathrm{~km}$, yielding a resolution of $1 \mathrm{~ns}$ with a phase stability of $200 \mathrm{ps}$.

Different solutions for station to data acquisition center communication (industrial miniPCs) and for slow control are available and being tested.

\subsection{Current prototype test setup}

The three Tunka-HiSCORe stations built so far have not yet reached the full planned setup. The PMTs used are EMI $9350 \mathrm{~KB}$, also in operation in the Tunka-133 array. In the future, we plan to use ET9352KB or Hamamatsu R (modified with 6 stages) PMTs [5]. The clipped-sum-trigger is not operational yet, thus far we have developed a summator board without clipping, the trigger being provided by one of three three possible methods: the Tunka-FADC trigger of the Tunka array, the onboard trigger of the WhiteRabbit system or the trigger of the DRS4 evaluation board which is used for readout. The DRS4 evaluation board [8] was developed by the
Paul Scherrer Institute and features a Domino Ring Sampler (DRS) 4 chip capable of 1024 bin sampling with 0.5-5 Gigasamples per second and a readout rate of up to $500 \mathrm{~Hz}$. The Evaluation board currently used has four channels plus one trigger input, but the DRS4 chip itself is capable of using up to eight channels plus trigger, which is an option considered for the custom DRS4 board which is currently under development.

\section{Results from the prototype station measurements}

Three prototype stations have been installed in October 2012, and were used for cross-calibration with the Tunka-133 Array (Fig. 1).

Different trigger measurements have been made to test background and minimum trigger threshold (see Fig. 2). Air-shower induced events are the main component of the trigger rate down to a trigger threshold of $70 \mathrm{mV}$ for the chosen station (dashed straight line for reference). Below, the night-sky background is responsible for the sharp increase of the trigger rate (dashed curved line for reference). The saturation of the measured raw trigger rate in the integrated spectrum (to be seen in Ref. [6]) lies at $20 \mathrm{~Hz}$, which gives us an estimation of the dead time of about $50 \mathrm{~ms}$.

\section{Outlook}

Nine stations have been deployed so far. The Tunka-HiSCORE engineering array will cover an area of about $1 \mathrm{~km}^{2}$ with about 60 stations, with the option of further expanding the array size to $10 \mathrm{~km}^{2}$ and more. Also, the final layout of the array is being under evaluation, finding the best compromise between sensitivity and the number of stations.

For improvement of the single stations, the use of UV wavelength shifter coating of the PMTs is explored. These shifters turn photons from 300 to $340 \mathrm{~nm}$ range to 360 to $400 \mathrm{~nm}$, thus increasing the trigger rate of a station by about two times.

Further analyses of the prototype data are underway (e.g. energy threshold and zenith angle acceptance).

\section{Acknowledgements}

This work was supported by the Russian Federation Ministry of Education and Science (G/C 14.518.11.7046, 14.B25.31.0010, 14.14 B37.21.0785, 14.B37.21.1294), the Russian Foundation for Basic Research (Grants 11-02-00409, 13-02-00214, 13-02-12095, 13-02-10001) and by the Helmholtz Association (Grant HRJRG303) and the Deutsche Forschungsgemeinschaft (Grant TL51-3).

\section{References}

[1] Hampf, Tluczykont, Horns, Nuclear Instruments and Methods in Physics Research A 137, 2012/13, doi:http://dx.doi.org/10.1016/j.nima.2013.02.016.

[2] Tluczykont, et al., Advances in Space Research 48 (2011) 1935.

[3] V. Prosin, in: Proceedings of Tunka-133: Results of 3 Year Operation, 2013.

[4] F. Berezhnev, et al., Tunka Collaboration, Nuclear Instruments and Methods in Physics Research A 692 (2012) 98.

[5] R. Wischnewski, et al., in: Proceedings of the ICRC 2013, ID1164, 2013.

[6] S. Epimakhov, et al., Components of the HiSCORE detector and prototype test results, in: Proceedings of the ICRC 2013, 2013.

[7] M. Brueckner, R. Wischnewski, in: First Results from the HiSCORE/Siberia WRsetup and Plans for CTA, 7th White Rabbit Workshop, Madrid, November 2012.

[8] Paul Scherrer Institute, Villigen, Switzerland, DRS4 Evaluation Board, 〈http:// www.psi.ch/drs/evaluation-board>. 